\title{
The estimation of the uncertainty associated with rating curves of the river Ivinhema in the state of Paraná/Brazil
}

\author{
Luiz Henrique Maldonado ${ }^{1,}{ }^{*}$, Daniel Firmo Kazay ${ }^{1}$, and Elio Emanuel Romero Lopez ${ }^{1}$ \\ ${ }^{1}$ Itaipu Binacional, Brazil and Paraguay
}

\begin{abstract}
The estimation of the uncertainty associated with stage-discharge relations is a challenge to the hydrologists. Bayesian inference with likelihood estimator is a promissory approach. The choice of the likelihood function has an important impact on the capability of the model to represent the residues. This paper aims evaluate two likelihood functions with DREAM algorithm to estimate specific non-unique stage-discharge rating curves: normal likelihood function and Laplace likelihood function. The result of BaRatin is also discussed. The MCMC of the DREAM and the BaRatin algorithm have been compared and its results seem consistent for the studied case. The Laplace likelihood function presented as good results as normal likelihood function for the residues. Other gauging stations should be evaluated to attend more general conclusions.
\end{abstract}

\section{Introduction}

The most common method for monitoring flow is to define it relation with the stage by a rating curve in a gauging station. This curve is usually determined empirically by means of periodic measurements of discharge and stage. The exponential form (equation 1) is widely used to represent the stage-discharge relation [1 - 3].

$$
Q=\alpha\left(h-h_{0}\right)^{n}
$$

where $\alpha$ is a coefficient, $\mathrm{h}_{0}$ is a cease-to-flow reference level, and $\mathrm{n}$ is an exponent. Considering the hydraulic controls and the reference regimes, the parameter $n$ should agree with typical theoretical values 5/3 (Manning-Strickler), 3/2 (rectangular weir) or $5 / 2$ (triangular weir). The parameter $\alpha$ also depends on the hydraulic controls and reference regimes, besides the geometry of the section, the declivity and the Strickler coefficient $[1,2]$.

The estimation of the uncertainty associated with stage-discharge is a challenge to the hydrologists. Bayesian inference is a particular promissory approach if the prior distributions of the parameters can be estimated with field data.

The choice of the likelihood function has an important impact on the capability of the model to represent the residues $[4,5]$. Therefore the likelihood function should be adapted with structural error of the model. As it is normal to observe higher structural uncertainty for higher flows, the structural model can be represented by a linearly-varying standard deviation: $\gamma_{1}+\gamma_{2} \mathrm{Q}[1,2]$. This approach is called heteroscedatic [4]. If we assume the error residuals to be normally distributed and uncorrelated then the likelihood function can be written as on equation 2.

$$
L\langle x \mid \tilde{Y}\rangle=\prod_{t=1}^{n} \frac{1}{\sqrt{2 \pi \widehat{\sigma}_{t}^{2}}} \exp \left[-\frac{1}{2}\left(\frac{\tilde{y}_{t}-y_{t}(x)}{\widehat{\sigma}_{t}}\right)^{2}\right]
$$

Where $\hat{\sigma}_{t}$ is $\gamma_{1}+\gamma_{2}$ Q. This formulation also can consider an unrealistic homoscedatic error, if $\hat{\sigma}_{t}$ is defined as a constant $\gamma_{1}$. Various others equation can be proposed to handle nontraditional error residual distributions [4]. The Laplace or double exponential distribution is a classical interesting likelihood function because it assumes a L1-norm of the error residuals, resulting in a loss of sensitivity to outliers (equation 3 ).

$$
L\langle x \mid \tilde{Y}\rangle=\prod_{t=1}^{n} \frac{1}{2 \widehat{\sigma}_{t}} \exp \left[-\frac{\left|\tilde{y}_{t}-y_{t}(x)\right|}{\widehat{\sigma}_{t}}\right]
$$

Where $\hat{\sigma}_{t}$ is $\gamma_{1}+\gamma_{2} \mathrm{Q}$.

After the definition of the prior distribution and likelihood function, Monte Carlo (MC) simulation methods are required to generate a sample of the posterior distribution. The DiffeRential Evolution Adaptive Metropolis (DREAM) algorithm

\footnotetext{
* Luiz Henrique Maldonado: 1hmaldo@itaipu.gov.br
} 
$[6,7]$ is a multi-chain Markov chain Monte Carlo (MCMC) simulation algorithm automatically tunes the scale and orientation of the proposal distribution en route to the target distribution, and exhibits excellent sampling efficiencies on complex, high-dimensional, and multi-modal target distributions. This algorithm is implemented on MATLAB scripts and has been used in many publications about hydrological processes [7].

BaRatin (BAyesianRATINg curve estimation [2, 13]), compose a statistical and hydraulic frameworks to estimate specific non-unique stage-discharge rating curves along with their uncertainties. The concept of hydraulic control to define the rating curve equations as well as the prior distributions of the parameters is presented. The algorithm uses the equation 2 as likelihood function with heteroscedatic residues and a combined Markov Chain Monte Carlo (MCMC) sampler with 4 chains to calculate the posterior probability density function [8].

This paper aims evaluate two likelihood functions (equations 1 and 2) with DREAM algorithm to estimate specific nonunique stage-discharge rating curves as modeled by BaRatin [13].

\section{Observations: uncertainty in individual gaugings}

The BaRatin framework also requires the analysis of individual gauging error, i.e., the observations provided to the Bayesian simulator [2]. Three kinds of errors should be highlighted to evaluate a stage-discharge relation: 1. measurement errors, combining instrumental errors, environmental errors, and spatial integration errors; 2. time integration error due to possible flow variability during the measurement period of time; 3 . stage-discharge bias due to non-reference flow conditions, such as the dynamic hysteresis effect $[1,2,3]$.

The uncertainty of mechanical current meter has been proposed on an ISO [9]. The quantification of the uncertainty of Doppler flow measurements has developed significantly in the last year. QRev [10] is an important framework developed to this purpose.

Otherwise, in extensive series of data, it is possible to lead with other kinds of error. For example, a mechanical current meter measurement can be affected by directional bias [11]. In the other hand, Doppler based current meter measurement can be biased by moving bottom [10,11]. Sometimes, in an ensemble of measurements, there is some without information about the effective occurrence of these sources of bias.

Changing the likelihood function is a way to deal with measurements that not respect the model of error residuals. The Laplace distribution can be a solution to minimize the importance of outliers. In this case, the outliers are the biased measurements.

At this work, to simplify the analysis, the errors of individual gauging are not considered (errors $=0$ ).

\section{Methodology}

\subsection{Study Case}

The study of case is a gauging station in the river Ivinhema in the state of Paraná/Brazil. The gauging station is located in straight segment of the river whose bed is composed basically of bedrock and sand. The catchment area is estimated in $32,000 \mathrm{~km}^{2}$.

Figure 1 presents the historic of bathymetries on the river section. It can be noted that the section is stable and can be modeled as a rectangle until the 3.8 meters of level with $200 \pm 30 \mathrm{~m}$ of base. At the section, the river flows in normal regime. The declivity of the river was estimated as $0.0005 \pm 0.0005 \mathrm{~m} / \mathrm{m}$ and the Strickler coefficient as $30 \pm 10$ $\mathrm{m}^{1 / 3} \cdot \mathrm{s}^{-1}$. The offset of the section control is set to $-0.8 \mathrm{~m} \pm 1 \mathrm{~m}$.

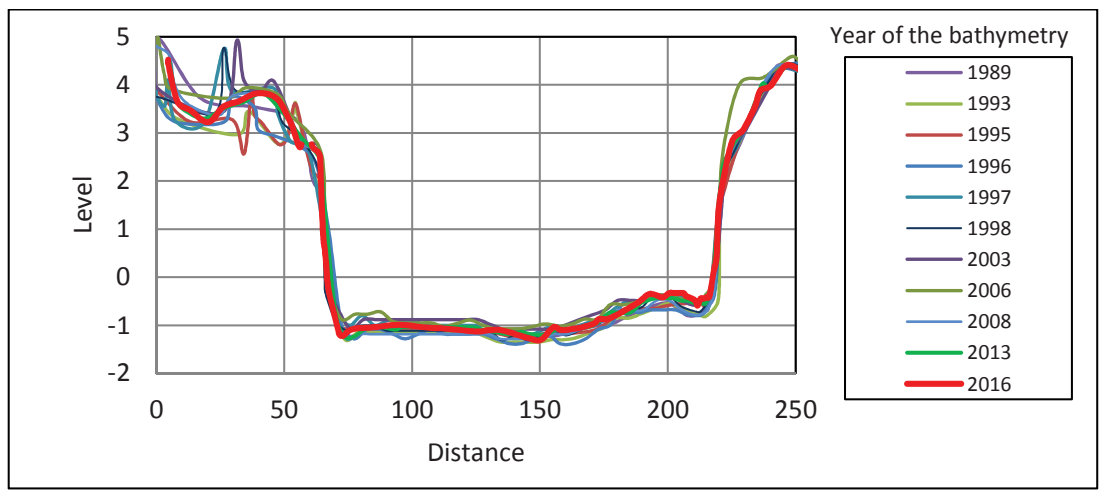

Fig. 1. Historic of bathymetries on the Ivinhema river section 


\subsection{Delimitation of the problem}

To simplify the problem, only the measurements of levels under 3.8 meters are considered. The period of data starts in 1972 and finishes in 2017 , covering 100 measurements. The maximum and minimum measured flows were $830 \mathrm{~m}^{3} . \mathrm{s}^{-1}$ and $114 \mathrm{~m}^{3} \cdot \mathrm{s}^{-1}$, respectively. The priors of the parameters with its minimum and maximum possible values are presented in Table 1. The first 48 measurement were used as calibration data and the last 52 were considered as validation data.

Two types of likelihood functions are evaluated: 1) equation 1 with heteroscedatic structural error, 2) equation 2 with heteroscedatic structural error. For each case, ten chains composed by 5,000 generations have been carried out.

The software BaRatinAGE v 2.0 [12] was used to compare the results.

The uncertainty associated with the results simulations was quantified by the confiability index.

Table 1.Priors of the parameters with its minimum and maximum possible values

\begin{tabular}{|c|c|c|c|c|}
\hline $\begin{array}{c}\text { Inferred } \\
\text { parameters }\end{array}$ & $\begin{array}{c}\text { Physical } \\
\text { parameters }\end{array}$ & Units & $\begin{array}{c}\text { Prior } \\
\text { Distributions }\end{array}$ & $\begin{array}{c}\text { Minimum and } \\
\text { maximum }\end{array}$ \\
\hline$\theta_{1}$ & $\mathrm{~A}$ & {$\left[\mathrm{~m}^{4 / 3} \cdot \mathrm{s}^{-1}\right]$} & Normal $(134,41.5)$ & $20-150$ \\
\hline$\theta_{2}$ & $\mathrm{~h}_{0}$ & {$[\mathrm{~m}]$} & Normal $(-0.8,0.5)$ & $-2-0$ \\
\hline$\theta_{3}$ & $\mathrm{~N}$ & {$[-]$} & Normal $(1.666,0.1)$ & $1.5-2$ \\
\hline $\mathrm{Y}_{1}$ & - & {$\left[\mathrm{m}^{3} \cdot \mathrm{s}^{-1}\right]$} & Uniform $\left(0,10^{6}\right)$ & - \\
\hline$\gamma_{2}$ & - & {$[-]$} & Uniform $\left(0,10^{6}\right)$ & - \\
\hline \multicolumn{4}{|r}{} \\
\hline
\end{tabular}

\section{Results}

To evaluate the possible differences between the MCMC of the DREAM and the BaRatin algorithm, the 95\% uncertainty band of the two methods were compared as showed in Figure 2. It is possible to remark that the methods are consistent.

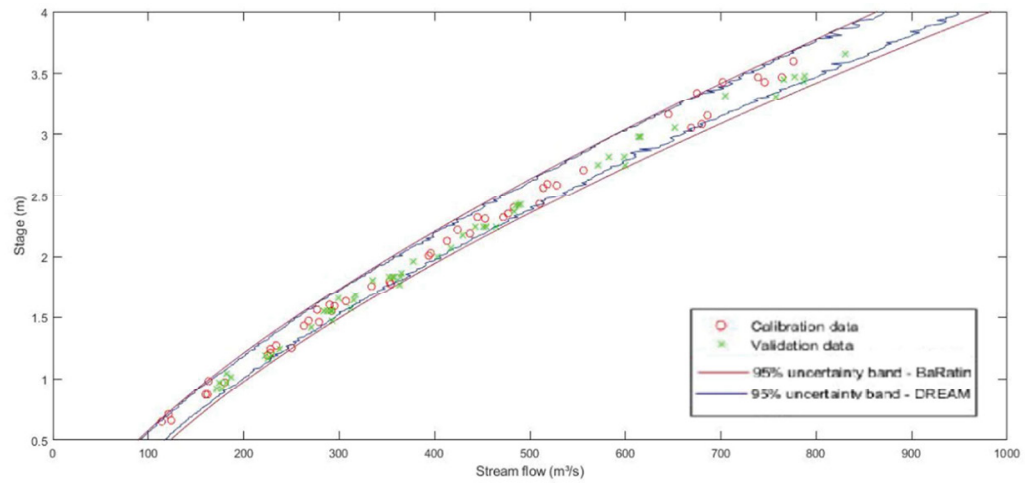

Fig. 2. Comparison of $95 \%$ uncertainty band for BaRatin and DREAM methods

The marginal distribution and bivariate scatter plot of the posterior samples are presented on figures 3 and 4 for each likelihood function. Finally the confiability index for calibration and validation data are disposed on figures 5 and 6 .

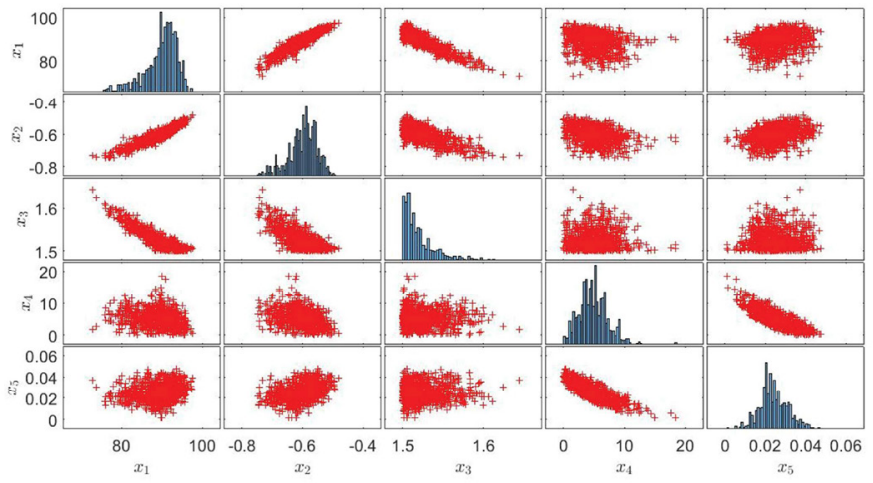

Fig. 3. Marginal distribution and bivariate scatter plot of the posterior samples with normal likelihood function for the residues 


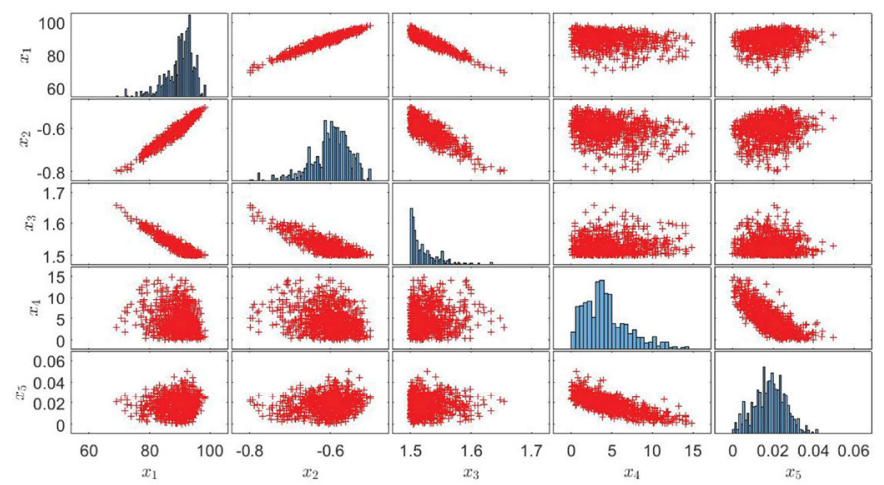

Fig. 4. Marginal distribution and bivariate scatter plot of the posterior samples with Laplace likelihood function for the residues

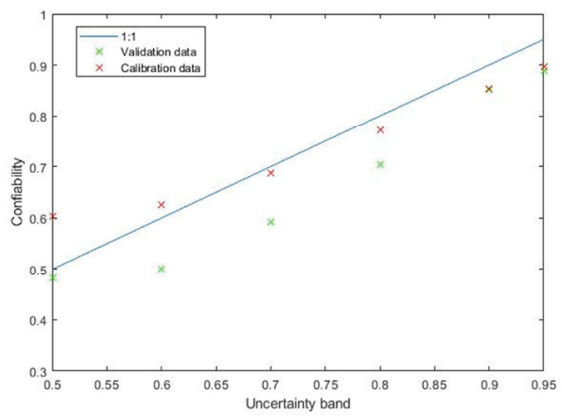

Fig. 5. Confiability index for calibration and validation data with normal likelihood function.

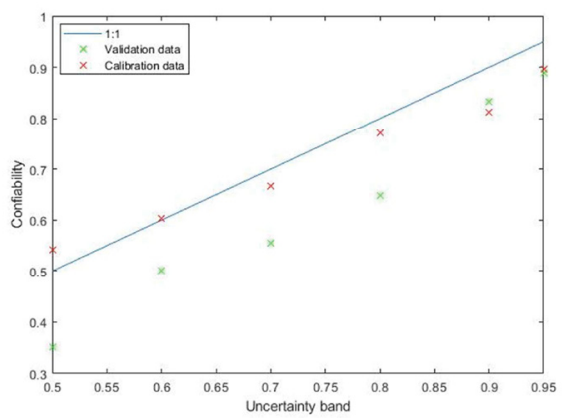

Fig. 6. Confiability index for calibration and validation data with Laplace likelihood function.

The Laplace distribution did not improve significantly the results. The punctual improvement of $95 \%$ validation band is compensated by the loss of quality of smaller validation bands.

\section{Conclusions}

The MCMC of the DREAM and the BaRatin algorithm have been compared and its results seem consistent for the studied case. The Laplace likelihood function presented as good results as normal likelihood function for the residues. It is important to highlight that just the low reach of gauging curve of a stable river has been considered in this paper. Other gauging stations should be evaluated to attend more general conclusions.

The individual gauging uncertainty can rule a major role on the results of the presented analysis and should be considered in future studies. However, the estimation of this kind of uncertainty still is a challenge for extensive series of data which different flow measured methods.

The authors thank all the field hydrology team for the day-a-day work that provides quality assured data and for this reason allowed the development of this paper. 


\section{References}

1. ValentinMansanarez. Non-unique stage-discharge relations: Bayesian analysis of complex rating curves and their uncertainties. (UniversitéGrenoble Alpes, 2016)

2. J. Le Coz, B. Renard, L. Bonnifait, F. Branger, R. LeBoursicaud.Combining hydraulic knowledge and uncertain gaugings in the estimation of hydrometric rating curves: A Bayesian approach.Journal of Hydrology, 509:573-587 (2014)

3. J. Le Coz. A literature review of methods for estimating the uncertainty associated with stage-discharge relations. Progress Report for the Word Meteorological Organization project «Assesment of the Performance of Flow Measurement Instrumentation and Techniques » (2014)

4. J. A. Vrugt. Markov chain Monte Carlo Simulation Using the DREAM Software Package: Theory, Concepts, and MATLAB Implementation. Environmental Modelling\& Software 75: 273-316 (2016)

5. D. Y. de Oliveira, P. L. B. Chaffe, J. H. Macedo Sá. Impacto da Escolha da Função de Verossimilhança na Estimativa de Incerteza e Inferência dos Parâmetros de um Modelo de Interceptação. In: Anais do XXII Simpósio Brasileiro de Recursos Hídricos(Porto Alegre: ABRH, 2013)

6. J.A. Vrugt, C.J.F. ter Braak, M.P. Clark, J.M. Hyman, B.A. Robinson. Treatment of input uncertainty in hydrologic modeling: Doing hydrology backward with Markov chain Monte Carlo simulation. Water Resources Research, vol. 44,W00B09, doi:10.1029/2007WR006720 (2008)

7. J.A. Vrugt, C.J.F. ter Braak, C.G.H. Diks, D. Higdon, B.A. Robinson, J.M. Hyman. Accelerating Markov chain Monte Carlo simulation by differential evolution with self-adaptive randomized subspace sampling.International Journal of Nonlinear Sciences and Numerical Simulation, vol.10, n. 3, pp. 273-290 (2009)

8. X. Sun. Regional frequency analysis of precipitation accounting for climate variability and change (Université de Grenoble, 2013)

9. International Standards Organization (ISO). Hydrometry - Measurements of liquid flow in open channels using current meters or floats. ISO 748 Technical Report. (Geneva, 1979)

10. D.S. Mueller.QRev-Software for computation and quality assurance of acoustic Doppler current profiler movingboat streamflow measurements-User's manual for version 2.8.U.S. Geological Survey Open-File Report 20161052, 50 p., http://dx.doi.org/10.3133/ofr20161052.

11. P. E. M. Gamaro, L. H. Maldonado, K. A. Lima. Comparison of Discharge Measurements Acquired With ADCP in a Moving Vessel and Fixed Vessel Techniques in Amazon River Basin. In: 1ed.London: Taylor \& Francis Group. (Org.). Rafael Murillo Muñoz. (Org.). River Flow (2012)

12. BaRatinAGE. BaRatin - BAyesian RATINg curve - Advanced Graphical Environment. https://forge.irstea.fr/projects/baratinage_v2 (2017)

13. Software BaRatin (C Irstea, Renard Benjamin, Bonnifait Laurent, Le Coz Jerome, Branger Flora, Kavetski Dmitri, $11 / 01 / 2017$. 